\title{
COVID-19 Propagation Model Based on Economic Development and Interventions
}

\author{
Fuzhong $\mathrm{Nian}^{1}$ (D) Yayong Shi $^{1} \cdot \mathrm{Jun}^{\mathrm{Cao}}{ }^{1}$
}

Accepted: 8 August 2021 / Published online: 18 August 2021

(C) The Author(s), under exclusive licence to Springer Science+Business Media, LLC, part of Springer Nature 2021

\begin{abstract}
In order to understand the influencing factors affecting the COVID-19 propagation, and analyze the development trend of the epidemic situation in the world, COVID-19 propagation model to simulate the COVID-19 propagation in the population is proposed in this paper. First of all, this paper analyzes the economic factors and interventions affecting the COVID-19 propagation in various different countries. Then, the touch number for COVID19 High-risk Population Dynamic Network in this paper was redefined, and it predicts and analyzes the development trend of the epidemic situation in different countries. The simulation data and the published confirmed data by the world health organization could fit well, which also verified the reliability of the model. Finally, this paper also analyzes the impact of public awareness of prevention on the control of the epidemic. The analysis shows that increasing the awareness of prevention, timely and early adoption of protective measures such as wearing masks, and reducing travel can greatly reduce the risk of infection and the outbreak scale.
\end{abstract}

Keywords Disease control · COVID-19 · Interventions · Propagation dynamics

\section{Introduction}

On December 12, 2019, pneumonia of unknown cause was discovered in Wuhan, Hubei Province, China. The World Health Organization refers to this epidemic as COVID-19 [1]. As of March 27, 2021, there were 126,700,364 confirmed cases and 2,779,741 deaths, and the number of confirmed cases is still increasing at an alarming rate. The outbreak of COVID-19 has serious socio-economic consequences, with excessive mortality and economic losses in some areas [2]. In the early stages of the pandemic, urban areas, especially large cities, were severely affected [3,4]. The evolving public discussion about the geographic impact of the pandemic requires a deeper understanding of the way the pandemic affects different vulnerable areas and requires different recovery plans.

Fuzhong Nian

gdnfz@lut.edu.cn

1 School of Computer and Communication, Lanzhou University of Technology, Lanzhou 730050, China 
During the outbreak of COVID-19, the differences in the speed and scale of infection between different regions attracted the research interest of scholars from many fields such as physics, biology, medicine, environmental science, and computer science [5-7]. First of all, some scholars have denied the mutation of the virus by calculating the basic reproduction number and analyzing the gene sequence of the virus in various regions of the world. Studies have shown that in the early stage of COVID-19 outbreak, the virus that broke out in various regions of the world did not mutate [8, 9]. It was the same virus. The virus has a moderate propagation capacity and can be prevented through reasonable epidemic prevention measures. Other scholars explained the differences in the scale of the outbreak of COVID-19 from the perspectives of population density, age structure, environmental factors, and epidemic prevention measures in different regions [10-20]. In addition to the factors of the city itself, there are also some social attributes that make some cities more susceptible to infection and larger outbreaks than other cities. As transportation hubs, these cities carry the role of transit in the flow of population. Geographical location, employment and industrial structure, population mobility, economic factors, etc [21-25]., will all affect the propagation of the virus in a region. In previous studies, we studied the time series data of confirmed patients in various regions of mainland China from January 16 to February 6 2020, and simulated the propagation of COVID-19 based on factors such as geographic location, economic conditions, and population movements, and the epidemic growth index was also proposed to measure the propagation process of the virus in each region [26]. Later, Jayson's research published on nature [27] also confirmed that there is a strong correlation between the propagation of COVID-19 in mainland China and the region's population mobility, economic conditions and other social attributes. Previous research has focused on the factors affecting the propagation of COVID-19 in mainland China. Because the epidemic continues to propagation, it has infected more than 100 million people in more than 200 countries around the world, and the number of infections is increasing. After the outbreak of COVID-19, all countries and regions in the world have been infected to varying degrees. Understanding the propagation trend of COVID-19 will better understand the propagation mechanism of COVID-19 and provide an important reference for epidemic prediction in other regions. In order to verify the impact of social attributes such as GDP on the cross-regional propagation of COVID-19 during the propagation of COVID-19, we conducted a time and space sequence analysis of the infection data of patients diagnosed with COVID-19. Time and space sequence analysis as a data analysis method have been widely used in epidemiological research, such as Zika virus, Ebola, SARS and so on [28-33]. In this research work, we analyzed the temporal and spatial sequence evolution data of confirmed cases in the United States, China, and Europe by combining social attributes, geographic distances, power law models, epidemic prevention measures, and public attitudes in different regions. Comparing the similarity of infection trends in different regions. Then, based on the characteristics of patients with COVID-19, we introduce a prediction model for the propagation of COVID-19 to simulate the propagation of COVID-19 in the population. The model divides the population into susceptible persons, latent persons, infected persons and recovered persons, and combines the time series infection data of confirmed patients to define the infection rate, latency rate and immunity rate of the model. The model introduces parameters such as medical resources and epidemic prevention measures in different regions, and predicts the propagation characteristics of the epidemic in the United Kingdom, Italy, Spain, and France. In addition, this article also discusses the impact of different epidemic prevention measures on the propagation of the virus. 
The rest of the paper is organized as follows. The characteristics of epidemic propagation in various countries with severe epidemics is analyzed in Sect. 2. The content of the experiment is in Sect. 3. In Section 4, the conclusion of the experiment are introduced respectively.

\section{Data Collection and Analysis}

\subsection{Data Collection}

In order to determine the factors influencing the propagation of COVID-19 in each country and region, this paper examines the factors that influence the propagation of COVID-19 in America, inland China, and Europe. Of all the countries infected by COVID-19 [34], America has the worst case. Until August 8, 2020, there have been 509,5524 confirmed cases in America. China serves as the first country where a mass infection has emerged and is finally under control. Studying the infection characteristics of COVID-19 in mainland China is important guidance for other countries that are suffering from the infection. In addition to studying infection characteristics in America and China, this paper examines COVID-19 infection characteristics in 43 European countries. Understanding the infection characteristics in each country will help us better understand how the COVID-19 propagations in various countries and regions, which will help us to fight against Epidemics to control the propagation of the virus.

America Infection data for America were obtained from the USA FACTS [35], They include the numbers of confirmed cases for 48 States, April 5,2021. GDP data for America were obtained from the U.S. Bureau of Economic Analysis [36].

China Infection data for China were obtained from the National Health Commission of the People's Republic of China and the Health Commission from other provinces [37]. They include the numbers of confirmed cases for 22 provinces, 5 autonomous regions, and 4 municipalities (Excluding Hong Kong, Macao, Taiwan, and Hainan), February 18, 2021. GDP data for China were obtained from the National Bureau of Statistics [38].

Europe Infection data for countries of Europe were obtained from the European Centre for Disease Prevention and Control (ECDC) [39]. They include the numbers of confirmed cases for 43 countries, April 1, 2021. GDP data for all the countries were obtained Statista [40].

\subsection{Analysis and Modeling}

Network structures used to represent social relationships when modeling the propagation of epidemics through a population include small-world networks, scale-free networks, etc [41-43]. Since the number of nodes in small-world networks and scale-free networks are fixed, their simulation results have certain limitations. In order to more realistically show the hidden relationships activated by the increase in the number of infected people, this article uses the high-risk population dynamic network [26] to simulate the social network during the propagation of COVID-19. The high-risk population dynamic network is a dynamic growth process, and the propagation rate of the epidemic largely depends on the scale of the high-risk dynamic network. With the increase of latent and infected individuals, neighbors around them also become high-risk groups, and there is a great possibility of being infected. The size of the network depends on 
the touch number $k$, which means that each infected node will bring $k$ new high-risk susceptible neighbors. When the newly joined neighbor nodes connect with nodes in the network, they follow the priority connection rule in the scale-free network. The touch number is affected by many factors. As an important social attribute of a region, the economic production activities of various regions will affect the number of contacts of the network $k$. A large number of economic production activities in developed regions will make people more frequent close contact, and frequent close contact will accelerate the propagation and propagation of the virus in the region. Figure 1a-c shows the GDP of America, China, and EU member states. Each country uses different colors to represent different levels of GDP. For example, in Fig. 1c, the red countries have the highest GDP, exceeding 1 trillion euros. The blue countries have the lowest GDP value, only below 200 billion. Figure 1d-f shows the infection status of America, China, and EU member states. Each country uses different colors to represent different levels of infection. The infection in red countries is the most serious, with more than 50,000 people infected. The blue country has the lowest level of infection, with less than 200 people infected. Comparing Fig. 1a, d, b, e, c, f, we can find that the GDP value has a strong correlation with the number of confirmed cases. Regions with high GDP have more confirmed cases, and regions with low GDP have fewer confirmed cases than other regions. In addition, when COVID-19 occurs, governments around the world will take different levels of intervention measures to curb the propagation of the virus in the country according to the development trend of the epidemic. The propagation of the virus in a country is obviously positively correlated with government interventions [44, 45]. Common intervention measures include traffic control, closing public places, adding temporary hospitals, adding medical equipment, and sending more medical staff to places where the epidemic is high. As an intuitive indicator, the case fatality rate reflects the effectiveness of government interventions. Taking effective intervention measures can effectively reduce the number of patients who died due to COVID-19. Therefore, we introduce the case fatality rate $f_{i}$ as a parameter to measure the effectiveness of intervention measures taken by governments around the world. Based on the above analysis and existing research, the touch number $k$ is redefined as follows.

(a)

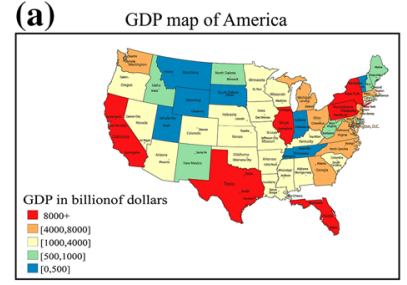

(d) Map of confirmed cases in America

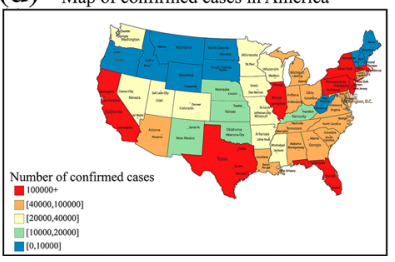

(b)

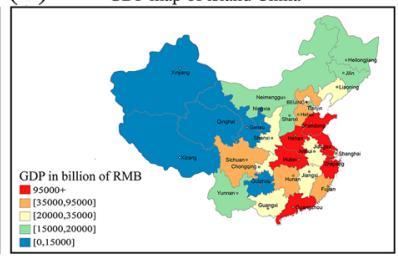

(e)

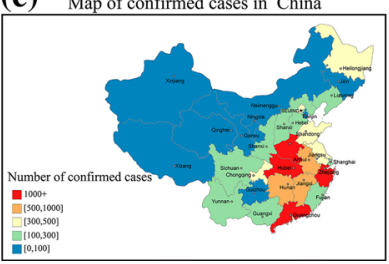

(c) GDP map of European Union

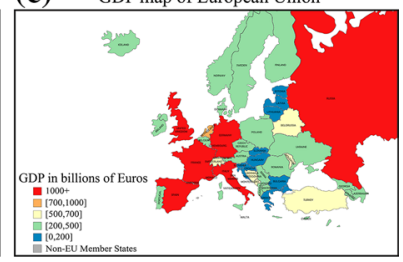

(f) Map of confirmed cases in European Union

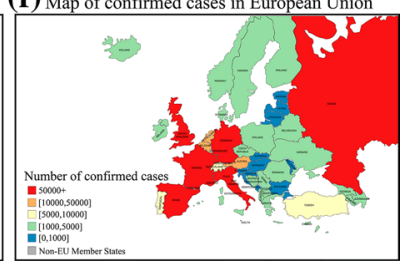

Fig. 1 The relationship between the GDP and the number of infected people in various countries and regions 


$$
\kappa_{i}=\frac{\mathrm{B}_{i} p_{i}}{\mathrm{~B}_{a} p_{a}}\left(1+f_{i}\right)
$$

Among, $\mathrm{B}_{i}$ and $\mathrm{B}_{a}$ refer to the gdp value of country $i$ and the average GDP value of each country, $p_{i}$ and $p_{a}$ refer to the population value of country $i$ and the average GDP value of each country, $f_{i}$ refers to the case fatality rate of country $i$. All values are normalized.

Combining with the time and space sequence evolution data of confirmed patients released by the World Health Organization, we simulate the propagation process of human infectious diseases by the COVID-19 propagation model as Fig. 2 [26].

The propagation rules of the COVID-19 propagation model are as follows.

1. The exposed and infected individuals can propagation the virus to susceptible individuals through close contact, and the propagation probability is $\beta$.

2. The latent probability of a exposed individual showing clinical symptoms after being infected and becoming an infected individual is $\varepsilon$.

3. Infected individual have a certain exit probability $\gamma$ to be quarantined, cured, or died to become the recovered individual, exit the infection circle, and no longer have the ability to propagation the virus.

The description of the differential equation based on the improved SEIR model is given as follows[26].

$$
\left\{\begin{array}{l}
\frac{d s(t)}{d t}=-\alpha(1-\varphi) s(e+i) \\
\frac{d e(t)}{d t}=\alpha(1-\varphi) s(e+i)-\frac{e}{1+e^{Q-T}} \\
\frac{d i(t)}{d t}=\frac{e}{1+e^{Q-T}}-\left(\frac{T-Q}{2 e Q}\right)^{2} i \\
\frac{d r(t)}{d t}=\left(\frac{T-Q}{2 e Q}\right)^{2} i
\end{array}\right.
$$

Among, $\alpha$ refers to the basic infection probability between individuals, which is a fixed value; $\varphi$ refers to the edge break rate of the node, that is the ratio of disconnected edges with surrounding nodes in order to protect itself from infection, $\varphi \in(0,1) . Q$ represents the average incubation period of the virus, and $T$ represents the length of time the patient has been sick. $s(t)$ stands for the proportion of susceptible individuals, $e(t)$ stands for the proportion of latent individuals, $i(t)$ stands for the proportion of infected individuals, $r(t)$ stands for the proportion of recover individuals.

Different countries have different cultural consciousness. People in different countries will adopt very different strategies to cope with the propagation of the disease. In order to verify the impact of different prevention strategies on the propagation of the epidemic, the following four prevention strategies were proposed.

Strategy 1. No prevention at all.

Strategy 2. Take precautions when more than half the neighborhood is protected.

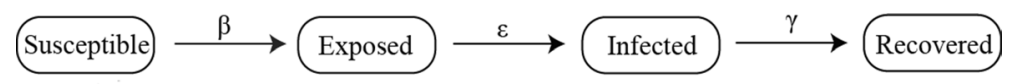

Fig. 2 Relationships between individual states 
Strategy 3. Take precautions when more than one the neighborhood is protected.

Strategy 4. Always take precautions.

\section{Experiment}

In this section, we studied the propagation characteristic of COVID-19 in more severely affected countries. Combining the basic attributes of each country (see Table 1), we have constructed the high-risk population dynamic network, and simulated the propagation of COVID-19 in the region through this network, as shown in the Fig. 3.

\subsection{The Outbreak Forecast}

Among the four countries shown in the Fig. 3, the number of confirmed patients in France and the United Kingdom are significantly higher than that in Italy and Spain. This is because the former has a larger population base and a more developed economy. The larger population base and economic conditions result in more frequent contacts with a relatively large number of people, which has accelerated the propagation of the virus in the country. It can be seen from the Fig. 3 that 60 days before the discovery of COVID-19, the number of infected individuals did not increase rapidly. At this time, COVID-19 is still in its infancy. If effective protective measures can be taken in time, even if there is no efficient medical care plan and vaccine, the local propagation of COVID-19 also can be prevented. However, the sporadic infections found locally did not attract the attention of these local governments and missed the best time to block them. When $60<t<100$, the number of infected people increases rapidly, which is caused by the weak protection awareness of the local government and people. The rapid increase in the number of infected people has attracted the attention of the local government and the people. Appropriate intervention measures and people's awareness of prevention have reduced the propagation of the virus in the local area. Therefore, when $100<t<260$, the number of active infections remains stable. But at this time, the fatality rate in each country is still at a high level, which shows that the propagation of COVID-19 has brought a heavy burden to the local government resources and medical system. Italy, France, and the United Kingdom have the highest case fatality rate of about 0.15 , which is very terrible. The stabilization of the number of infected individuals confuses the public's audiovisual, and the shortage of medical resources makes some infected individuals unable to be isolated and treated, which will accelerate the propagation of COVID-19 in the local area. When $t>260$, the number of infected individuals in the four countries increased rapidly, and maintained a high growth rate. As of April 15,2021 , the number of infected individuals has exceeded 3,000,000. At this time, taking

Table 1 Parameter setting in simulation and experiment

\begin{tabular}{lllllll}
\hline Country & $B$ & $p$ & $\varphi_{t<60}$ & $\varphi_{60<t<100}$ & $\varphi_{100<t<260}$ & $\varphi_{t>260}$ \\
\hline United Kingdom & 1 & 0.99 & 0 & 0.5 & 0.2 & 0.35 \\
France & 0.96 & 1 & 0 & 0.5 & 0.2 & 0.35 \\
Italy & 0.70 & 0.89 & 0 & 0.5 & 0.2 & 0.35 \\
Spain & 0.52 & 0.70 & 0 & 0.5 & 0.2 & 0.35 \\
\hline
\end{tabular}


(a)

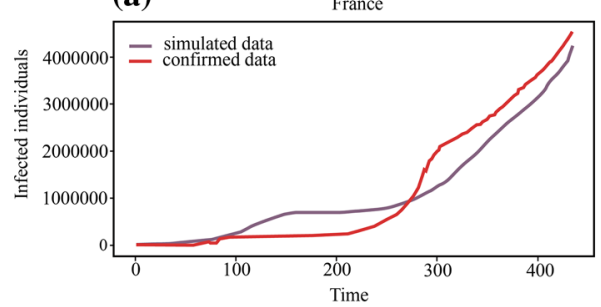

(c)

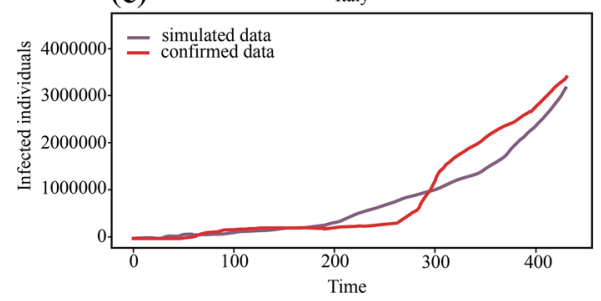

(b)

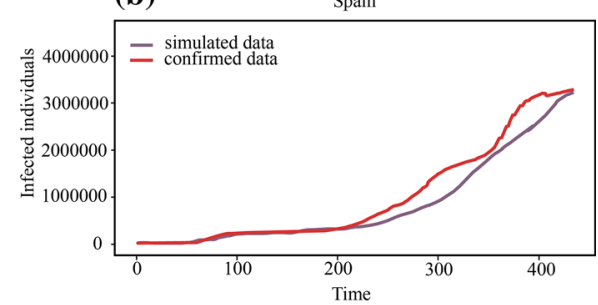

(d)

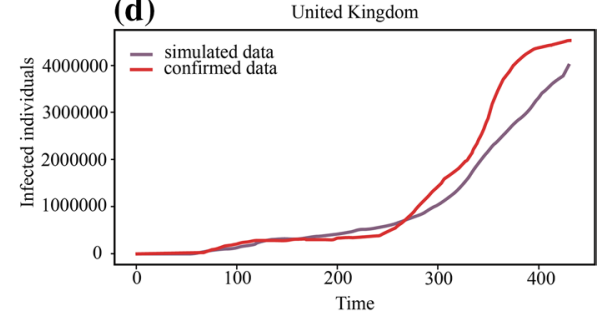

(e)

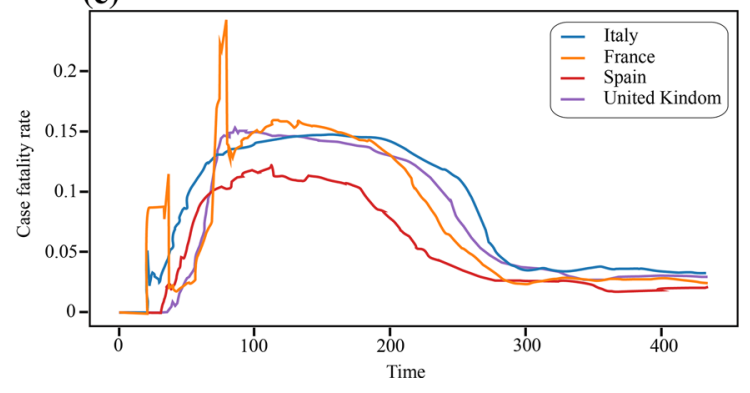

Fig. 3 Infection curve

some social measures can delay the propagation of the virus to a certain extent, but it has been unable to completely isolate the propagation of the virus. The social cost of isolation measures is very huge. At this time, COVID-19 has evolved into an infectious disease that has coexisted with humans for a long time, and its types have also become three types: asymptomatic infections, mild infections, and severe infections. This has reduced the fatality rate to a certain extent, but the large number of infected people makes it more difficult for the government to screen, and it is impossible to control the propagation of the virus through short-term and small-scale social measures.

\subsection{The Impact of Protective Strategies on the Propagation of COVID-19}

A variety of factors affect the COVID-19 propagation, among them, public awareness of prevention is an important factor. The public's awareness of the outbreak will influence their prevention strategies. Individuals can inhibit the the COVID-19 propagation by raising awareness and strengthening protective measures. In order to verify the impact of different prevention awareness on the epidemic propagation, in this paper, four different 
Fig. 4 Individual's State Relationship

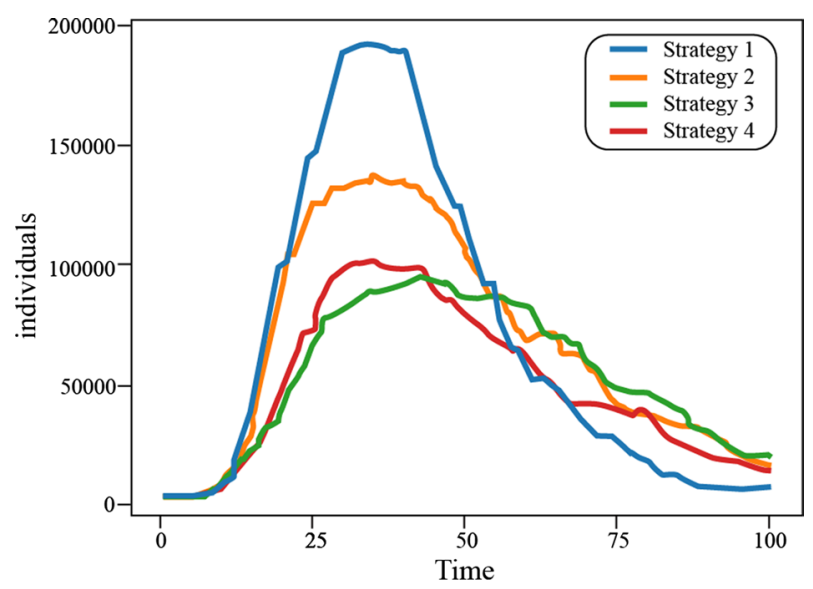

prevention strategies were used to simulate the impact of individuals' awareness of the epidemic on the COVID-19 propagation.

As shown in Fig. 4, When all the individuals in the network adopt strategy 1(no prevention at all), the COVID-19 carrier's number is around 200,000, much bigger than other strategies. When all the individuals in the network adopt strategy 2(take precautions when more than half the neighborhood is protected), the COVID-19 carrier's number is around 130,000 , lower than strategy 1 . Strategy 3 (take precautions when more than one neighborhood is protected) and strategy 4(always take precautions) had roughly the same impact on the COVID-19 carrier's number. Comparing with strategy 1 and strategy 2, the COVID-19 carrier's number was greatly reduced to 100,000. From the above experiments, it can be seen that the awareness of the epidemic determines the COVID-19 carrier's number, and negative and slack attitudes can accelerate the COVID-19 propagation. When the outbreak occurs, although there are no confirmed patients around, timely and early adoption of protective measures such as wearing masks and reducing travel can greatly reduce the risk of infection and greatly reduce the outbreak scale.

\section{Conclusion}

on the analysis of the countries with severe epidemic diseases outbreak, the high-risk population dynamic network was proposed. Combining the economic, medical conditions, and the different intervention measures of different countries, the corresponding initial values of the parameters in the network were set, and then use the COVID-19 spread model to simulate the development trend of severely affected countries. The simulation shows that the epidemic development in each country is affected by economic conditions, and interventions. Good economic conditions will accelerate the propagation of the epidemic, but adequate medical conditions and appropriate interventions will significantly reduce the size of the COVID-19 carrier and shorten the time of its propagation. The paper also discusses the impact of public awareness on the epidemic propagation. The experiment shows that increasing the awareness of prevention, timely and early adoption of protective measures such as wearing masks and reducing travel can greatly reduce the risk of infection and greatly reduce the outbreak scale. 
Acknowledgements This research is supported by the National Natural Science Foundation of China (No. 61863025), Program for International S \& T Cooperation Projects of Gansu province (No. 144WCGA166), Program for Longyuan Young Innovation Talents and the Doctoral Foundation of LUT.

\section{References}

1. Sohrabi, C., Alsafi, Z., O’Neill, N., Khan, M., \& Agha, R. (2020). Corrigendum to world health organization declares global emergency: a review of the 2019 novel coronavirus (covid-19). International Journal of Surgery, 76, 71-76.

2. Streeck, H., Schulte, B., Kümmerer, B. M., et al. (2020). Infection fatality rate of SARS-CoV2 in a super-spreading event in Germany. Nature Communications, 11, 5829.

3. Rendeiro, A. F., Ravichandran, H., Bram, Y., et al. (2021). The spatial landscape of lung pathology during COVID-19 progression. Nature. https://doi.org/10.1038/s41586-021-03475-6.

4. James, Nick, Menzies, Max, \& Radchenko, Peter. (2021). COVID-19 second wave mortality in Europe and the United States. Chaos: An Interdisciplinary Journal of Nonlinear Science, 31, 031105.

5. Rozhnova, G., van Dorp, C. H., Bruijning-Verhagen, P., et al. (2021). Model-based evaluation of school- and non-school-related measures to control the COVID-19 pandemic. Nature Communications, 12, 1614.

6. Willem, L., Abrams, S., Libin, P. J. K., et al. (2021). The impact of contact tracing and household bubbles on deconfinement strategies for COVID-19. Nature Communications, 12, 1524.

7. Manchein, C., Brugnago, E. L., da Silva, R. M., Mendes, C. F. O., \& Beims, M. W. (2020). Strong correlations between power-law growth of COVID-19 in four continents and the inefficiency of soft quarantine strategies. Chaos: An Interdisciplinary Journal of Nonlinear Science, 30, 041102.

8. Zhao, Shi, Lin, Qianyin, Ran, Jinjun, Salihu, S., et al. (2020). Preliminary estimation of the basic reproduction number of novel coronavirus (2019-nCoV) in China, from 2019 to 2020: A data-driven analysis in the early phase of the outbreak. International Journal of Infectious Diseases, 92, 214-217.

9. Neal, Peter, \& Theparod, Thitiya. (2019). The basic reproduction number, R0, in structured populations. Mathematical Biosciences, 315, 108224.

10. Fernández, Daniel, Giné-Vázquez, Iago, Liu, Ivy, Yucel, Recai, et al. (2021). Are environmental pollution and biodiversity levels associated to the spread and mortality of COVID-19?. A four-month global analysis. Environmental Pollution, 271, 116326.

11. Giordano, G., Colaneri, M., Di Filippo, A., et al. (2021). Modeling vaccination rollouts, SARS-CoV-2 variants and the requirement for non-pharmaceutical interventions in Italy. Nature Medicine. https:// doi.org/10.1038/s41591-021-01334-5.

12. Woolcott, O. O., \& Castilla-Bancayán, J. P. (2021). The effect of age on the association between diabetes and mortality in adult patients with COVID-19 in Mexico. Scientific Reports, 11, 8386.

13. Ingram, M., Zahabian, A., \& Hur, C. (2021). Prediction of COVID-19 Social Distancing Adherence (SoDA) on the United States county-level. Humanities and Social Sciences Communications, 8, 87.

14. Zietz, M., Zucker, J., \& Tatonetti, N. P. (2020). Associations between blood type and COVID-19 infection, intubation, and death. Nature Communications, 11, 5761.

15. Huang, B., Wang, J., Cai, J., et al. (2020). Integrated vaccination and physical distancing interventions to prevent future COVID-19 waves in Chinese cities. Nature Human Behaviour. https://doi.org/ 10.1038/s41562-021-01063-2.

16. Pinto Neto, O., Kennedy, D. M., Reis, J. C., et al. (2020). Mathematical model of COVID-19 intervention scenarios for São Paulo-Brazil. Nature Communications, 12, 418.

17. akahashi, T., Ellingson, M. K., Wong, P., et al. (2020). Sex differences in immune responses that underlie COVID-19 disease outcomes. Nature, 588, 315-320.

18. Wells, C. R., Townsend, J. P., Pandey, A., et al. (2021). Optimal COVID-19 quarantine and testing strategies. Nature Communications, 12, 356.

19. Behring, Brandon M.., \& Rizzo, Alessandro. (2021). How adherence to public health measures shapes epidemic spreading: A temporal network model. Chaos: An Interdisciplinary Journal of Nonlinear Science, 31, 043005.

20. Nande, A., Sheen, J., Walters, E. L., et al. (2021). The effect of eviction moratoria on the transmission of SARS-CoV-2. Nature Communications, 12, 2274.

21. Haug, N., Geyrhofer, L., Londei, A., et al. (2020). Ranking the effectiveness of worldwide COVID-19 government interventions. Nature Human Behaviour, 4, 1303-1312.

22. Rendeiro, A. F., Ravichandran, H., Bram, Y., et al. (2021). The spatial landscape of lung pathology during COVID-19 progression. Nature., 6, 564-569. 
23. Vadrevu, K. P., Eaturu, A., Biswas, S., et al. (2020). Spatial and temporal variations of air pollution over 41 cities of India during the COVID-19 lockdown period. Scientific Reports, 10, 16574.

24. Solimini, A., Filipponi, F., Fegatelli, D. A., et al. (2021). A global association between Covid-19 cases and airborne particulate matter at regional level. Scientific Reports, 11, 6256.

25. Middya, A. I., \& Roy, S. (2021). Geographically varying relationships of COVID-19 mortality with different factors in India. Scientific Reports, 11, 7890.

26. Yayong, S. H. I., Fuzhong, N. I. A. N., Jinshuo, L. I. U., et al. (2020). Propagation dynamics of COVID-19 in high-risk population dynamic network. Control Theory \& Applications, 37, 461-468.

27. Jia, J. S., Lu, X., Yuan, Y., et al. (2020). Population flow drives spatio-temporal distribution of COVID19 in China. Nature, 582, 389-394.

28. Graham, R., Donaldson, E., \& Baric, R. (2013). A decade after SARS: strategies for controlling emerging coronaviruses. Nature Reviews Microbiology, 11, 836-848.

29. Diamond, B. (2003). SARS spreads new outlook on quarantine models. Nature Medicine, 9, 1441.

30. Madelain, V., Baize, S., Jacquot, F., et al. (2018). Ebola viral dynamics in nonhuman primates provides insights into virus immuno-pathogenesis and antiviral strategies. Nature Communications, 9, 4013.

31. Dellicour, S., Baele, G., Dudas, G., et al. (2018). Phylodynamic assessment of intervention strategies for the West African Ebola virus outbreak. Nature Communications, 9, 2222.

32. Aris-Brosou, S., Ibeh, N., \& Noël, J. (2017). Viral outbreaks involve destabilized evolutionary networks: Evidence from Ebola. Influenza and Zika Scientific Reports, 7, 11881.

33. Xia, Z. Q., Wang, S. F., Li, S. L., et al. (2015). Modeling the transmission dynamics of Ebola virus disease in Liberia. Scientific Reports, 5, 13857.

34. Max, R., Hannah, R., Esteban, O. Joe, H. (2020). Coronavirus Pandemic (COVID-19). Published online at OurWorldInData.org. Retrieved from: https://ourworldindata.org/coronavirus [Online Resource].

35. USAFACTS. (2020). Coronavirus Locations: COVID-19 Map by County and State. Coronavirus Stats \& Data. https://usafacts.org/visualizations/coronavirus-covid-19-spread.

36. U.S. Bureau of Economic Analysis. (2020). Local Area Gross Domestic Product, 2018. GDP by County, Metro, and Other Areas. https://www.bea.gov/data/gdp/gdp-county-metro-and-other-areas.

37. National Health Commission of the People's Republic of China. (2020).Outbreak notification. Novel coronavirus pneumonia outbreak prevention and control. http://www.nhc.gov.cn/xcs/yqtb/list_gzbd. shtml.

38. National Bureau of Statistics of China. (2019). Gross Reginoal Product (2018) . in CHINA STATISTICAL YEARBOOK-2019, (9th ed., Vol. 3). Beijing, China: China Statistics Press.

39. European Centre for Disease Prevention and Control. (2020). COVID-19-geographic-disbtributionworldwide. [Online]. https://www.ecdc.europa.eu/en/publications-data/download-todays-data-geogr aphic-distribution-covid-19-cases-worldwide.

40. Statista. (2020). Gross domestic product (GDP) at current market prices of selected European countries in 2018 (in billion euros). Economy. https://www.statista.com/statistics/685925/gdp-of-europeancountries/.

41. Munday, J. D., Sherratt, K., Meakin, S., et al. (2021). Implications of the school-household network structure on SARS-CoV-2 transmission under school reopening strategies in England. Nature Communications, 12, 1942.

42. Fulker, Z., Forber, P., Smead, R., et al. (2021). Spite is contagious in dynamic networks. Nature Communications, 12, 260.

43. Aral, S., \& Nicolaides, C. (2017). Exercise contagion in a global social network. Nature Communications, 8, 14753.

44. Tian, L., Li, X., Qi, F., et al. (2021). Harnessing peak transmission around symptom onset for nonpharmaceutical intervention and containment of the COVID-19 pandemic. Nature Communications, 12, 1147.

45. Ragonnet-Cronin, M., Boyd, O., Geidelberg, L., et al. (2021). Genetic evidence for the association between COVID-19 epidemic severity and timing of non-pharmaceutical interventions. Nature Communications, 12, 2188.

Publisher's Note Springer Nature remains neutral with regard to jurisdictional claims in published maps and institutional affiliations. 


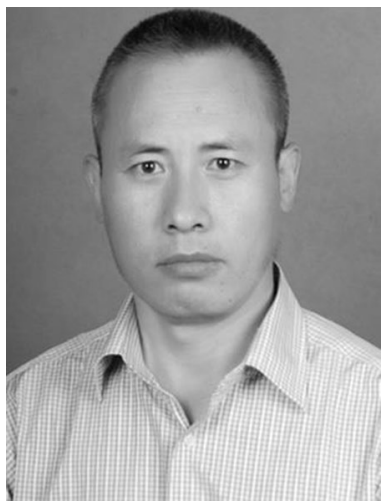

Fuzhong Nian received the B.S. degree in engineering from Northwest Normal University (department of Physics), LanZhou, China, in 1998; the M.S. degree in engineering from Gansu University of Technology, Lanzhou, China, in 2004; and the Ph.D. degree in engineering from Dalian University of Technology, Dalian, China, in 2011. He is interested in research at the intersection of mathematical modeling, network science, and control theory with application to biological, social and chaotic network.

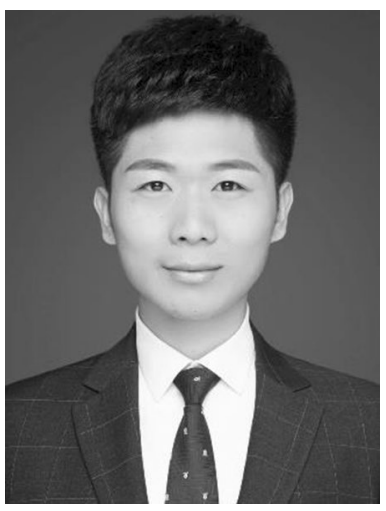

Yayong Shi received the B.S. degree in mechanical engineering from Qilu University of Technology, Jinan, China in 2017, and is currently pursuing the M.S. degree in computer technology at Lanzhou University of Technology, Lanzhou, China. His main research interests include the modeling and analysis of complex networks, with applications in epidemic spreading and social networks.

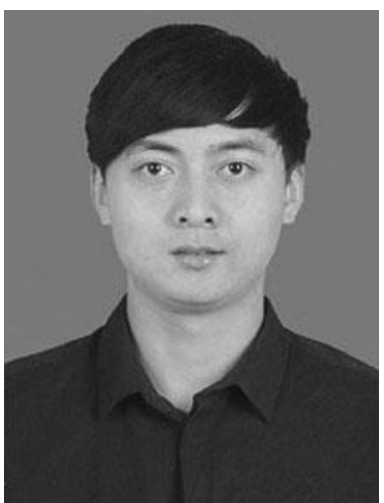

Jun Cao received the B.S. degree in mechanical engineering from Lanzhou Polytechnical College, Jinan, China in 2017, and is currently pursuing the M.S. degree in computer technology at Lanzhou University of Technology, Lanzhou, China. His main research interests include the modeling and analysis of complex networks, with applications in epidemic spreading and social networks, intelligent data analysis 